\section{GP114 A 5 YEAR AUDIT OF DENTAL CARIES EXPERIENCE OF 5 YEAR OLD CHILDREN ATTENDING THE DUBLIN CLEFT CENTRE}

${ }^{1}$ Eleanor Mc Govern*, ${ }^{2,3}$ Kirsten FitzGerald. 'Temple Street Children's University Hospital, Dublin, Ireland; ${ }^{2}$ Our Lady's Children Hospital, Crumlin, Dublin, Ireland; ${ }^{3}$ Dublin Dental University Hospital, Dublin, Ireland

10.1136/archdischild-2019-epa.179

It is well recognised that children with a cleft of the lip, palate or both, are at increased risk of and from dental caries. Children attending the Dublin Cleft Centre for cleft care are referred by their first birthday to community/primary care dental services for prevention of dental disease. These children also attend the paediatric specialist dental service at their tertiary hospital while attending multidisciplinary team clinics, and/ or if advanced dental treatment is required.

As well as joint review and planning clinics, the service also runs audit clinics throughout the year. A measure of dental caries is recorded (by calibrated specialists and consultants in Paediatric Dentistry) for the dental component of the multidisciplinary audit. Dental caries is recorded using the $\mathrm{dmft}$ index (decayed, missing, filled, teeth). We report on the dental caries experience of five year old children (born 2007, 2008, 2009, 2010 and 2011) with a cleft of the lip, palate or both.

The mean dmft of the 277 children included in the audit was 3.25. Excluding children who were caries free, the mean $\mathrm{dmft}$ was 5.4. The mean $\mathrm{dmft}$ (per year cohort) has reduced from 3.8 for children born in 2007 to 2.1 for children born in 2011. The percentage of children who are caries free $(\mathrm{dmft}=0)$ has increased from $32 \%$ of children born in 2007 to $66 \%$ of children born in 2011 . The percentage of children who are dentally fit $(\mathrm{d}=0)$ has increased from $46 \%$ of children born in 2007 to $77 \%$ of children born in 2011 .

It is clear that the prevalence of dental caries (mean $\mathrm{dmft}$ ) has reduced over the 5 year timeframe. There has also been a significant increase in the number of children who are caries free $(\mathrm{dmft}=0)$, and in the number of children who are dentally fit $(\mathrm{d}=0)$. This improvement in oral health may be as a result of improved tertiary care dental service resources and/or improved communication and follow up with the primary care dental services.

\section{GP115 TODDLERS, TEENS AND EVERYTHING IN BETWEEN. SHOULD ALL CHILDREN BE ADMITTED TO THE SAME WARD?}

Rachel Mullaly*, Elena Nechita, Mags Clancy, Dara Gallagher. Sligo University Hospital, Sligo, Ireland

10.1136/archdischild-2019-epa.180

Aims To define the adolescent population admitted to the Paediatric Ward in Sligo University Hospital. To determine the attitude of adolescents and all parents towards room sharing between children of different age groups and to examine the use of recreational facilities on the Paediatric Ward.

Methods A retrospective review of patients admitted to the Paediatric Ward from October 2018 to December 2018 was performed. We defined adolescents as patients $\geq 13$ years of age (yoa). Three separate questionnaires were designed for three defined groups (adolescents aged $\geq 13$ yoa, parents of adolescents $\geq 13$ yoa, parents of children $<13$ yoa).
Questionnaires were distributed to each of these three groups admitted to the Paediatric Ward.

Results 529 patients were identified. 158, 192 and 179 in October, November and December respectively. In October 27/158 (17\%) of admissions were adolescents. In November $36 / 192(19 \%)$ of admissions were adolescents. In December $16 / 179(9 \%)$ of admissions were adolescents. During time of questionnaire distribution participants were admitted to single rooms and shared rooms with patient's ages ranging from 4 months to 17 yoa. All those surveyed, including patients $>16$ yoa (our normal age cut-off for admission to Paediatric Ward) expected to be admitted to the 'Children's Ward'. Most adolescents and their parents were 'not bothered' by the ages of children sharing the same room as them. Most adolescents and their parents claimed that they would be happy to share a room with older adolescents, i.e. 16 to 17-year olds. However, parents of younger children (<13yoa) generally did mind adolescents sharing the same room as their children and would prefer if children within the same age bracket only were sharing the room. No adolescents used the Playroom during their stay. More children $<13$ yoa tended to use the Playroom. Most of the adolescents and their parents felt that their recreational needs were not met. Parents generally advocated for the presence of a 'Teen Den' as opposed to Playroom. All adolescents either preferred the idea of a Teen Den or were indifferent towards it. Some parents of children $<13$ yoa were 'bothered' that adolescents could have use of the same Playroom as their children.

Conclusion The establishment of an adolescent-specific recreational area for adolescents admitted to the Paediatric Ward in Sligo University Hospital should be established to cater for their recreational needs and well-being. Adolescents on the Paediatric Ward should have different accommodation to younger children.

\section{GP116 APPROACH TO MANAGING SPASTICITY IN HEREDITARY SPASTIC PARAPLEGIA}

Rushna Raza*, Raj Lodh. Leeds Teaching Hospital NHS Trust, Leeds, UK

\subsection{6/archdischild-2019-epa.181}

Aim We aim to provide a guidance in the management of spasticity in children with Hereditary Spastic Paraplegia (HSP). Introduction Hereditary spastic paraplegia is a group of clinically and genetically diverse disorders that result in progressive and generally severe lower extremity weakness and spasticity. Spinal and brain MRI and exome sequencing are the most useful investigations for diagnosing HSP. There is a lack of evidence base and guidance for the use of anti-spasticity drugs and uncertainty about the place of other modalities such as intrathecal baclofen (ITB), selective dorsal Rhizotomy (SDR) and botulinum toxin in HSP.

Method A guideline was prepared based on consultation with experts and regional specialist practice (Level D Evidence) supported by Level A-C evidence for individual treatment options. The guideline includes mode of action, dosage suggestions, side effect profile and cautions for the treatment modalities and summarises key management steps in a flow chart.

Management of spasticity Management of spasticity in HSP is based on identifying clear and realistic goals in the following three areas: improving mobility, increasing range of motion and relieving spasticity. These goals can be achieved through a 